\title{
Osteoblasts Display Different Responsiveness to TRAIL-Induced Apoptosis During Their Differentiation Process
}

\author{
Giacomina Brunetti - Angela Oranger - Claudia Carbone · Giorgio Mori • \\ Francesca Rita Sardone - Claudio Mori • Monica Celi • Maria Felicia Faienza • \\ Umberto Tarantino $\cdot$ Alberta Zallone $\cdot$ Maria Grano $\cdot$ Silvia Colucci
}

(C) Springer Science+Business Media New York 2013

\begin{abstract}
Apoptosis can occur throughout the life span of osteoblasts (OBs), beginning from the early stages of differentiation and continuing throughout all stages of their working life. Here, we investigated the effects of tumor necrosis factor (TNF)-related apoptosis-inducing ligand (TRAIL) on normal human OBs showing for the first time that the expression of TRAIL receptors is modulated during OB differentiation. In particular, the TRAIL receptor ratio was in favor of the deaths because of the low expression of DcR2 in undifferentiated OBs, differently it was shifted toward the decoys in differentiated ones. Undifferentiated OBs treated with TRAIL showed reduced
\end{abstract}

Electronic supplementary material The online version of this article (doi:10.1007/s12013-013-9616-6) contains supplementary material, which is available to authorized users.

G. Brunetti - A. Oranger - C. Carbone - F. R. Sardone ·

A. Zallone - M. Grano $\cdot$ S. Colucci $(\bowtie)$

Section of Human Anatomy and Histology - R. Amprino, Department of Basic Medical Sciences, Neuroscience and Sense Organs, Medical School, University of Bari, Piazza Giulio Cesare 11, 70124 Bari, Italy

e-mail: silviaconcetta.colucci@uniba.it

G. Mori

Department of Clinical and Experimental Medicine,

University of Foggia, Foggia, Italy

C. Mori

I Orthopaedic and Traumatology Unit, General Hospital,

University of Bari, Bari, Italy

M. Celi · U. Tarantino

Orthopaedics and Traumatology, PTV Foundation, University of Tor Vergata, Rome, Italy

M. F. Faienza

Department of Biomedical Sciences and Human Oncology,

University of Bari, Bari, Italy cell viability, whereas differentiated OBs displayed TRAIL resistance. The OB sensitiveness to TRAIL was due to the up-regulation of DR5 and the down-regulation of DcR2. The main death receptor involved in TRAIL-reduced OB viability was DR5 as demonstrated by the rescue of cell viability observed in the presence of anti-DR5 neutralizing antibody. Besides the ratio of TRAIL receptors, the sensitivity of undifferentiated OBs to TRAIL-cytotoxic effect was also associated with low mRNA levels of intracellular anti-apoptotic proteins, such as cFLIP, the activation of caspase- 8 and -3 , as well as the DNA fragmentation. This study suggests that apoptotic effect exerted by TRAIL/ TRAIL-receptor system on normal human OB is strictly dependent upon cell differentiation status.

Keywords Osteoblast - TRAIL · Apoptosis - TRAIL receptor $\cdot$ DcR2 $\cdot$ c-FLIP

\section{Introduction}

Controlled cell death by apoptosis is recognized as an important component of embryogenesis and tissue morphogenesis, and, in adult skeletons it contributes substantially to physiological bone turnover, repair, and regeneration [1-3]. It is now generally accepted that the number of osteoblasts (OBs), the bone-forming cells, plays a critical role in bone turnover, and all major regulators of bone contribute to the regulation of OBs life span by modulating apoptosis [4].

In particular, apoptosis of OBs was reported during all physiological periods within their life span, beginning at the early stages of differentiation and continuing throughout the working life of this cell type [4]. It is believed that this process occurs in order to make space for advancing 
bone formation within the skeletal system. This was supported by the demonstration that once bone formation is completed, $\sim 50-70 \%$ of the OBs originally assembled at remodeling sites undergo apoptosis [4]. As apoptosis of bone cells is a rapid process and cannot be assessed in vivo during bone turnover in a normal adult organism [5], expression and function of apoptotic molecules, belonging to tumor necrosis factor (TNF) ligand and receptor families, have been investigated on bone cells in vitro, with controversial results from different experimental models and methods [1, 6, 7]. Among the members of the TNF family, some authors have examined the sensitivity of OBs to TNF-related apoptosis-inducing ligand (TRAIL), but contradictory results have also emerged from these studies [8-14].

TRAIL is a type II transmembrane protein that induces apoptosis through interactions with its two death domaincontaining receptors, namely, DR4 and DR5 [15, 16]. Ligand-dependent activation of DR4 and DR5 involves receptor multimerization, with subsequent recruitment of Fas-associated protein with death domain that engages the initiator protease pro-caspase-8, via an interaction of their respective death effector domain [17]. TRAIL-activated caspase- 8 subsequently leads to activation of downstream executioner caspases, including caspase- 3 and -7 , resulting in the cleavage of cellular substrates and ultimately cell death $[18,19]$. However, DR4 and DR5 activity can be antagonized by the three so-called "decoy" receptor for TRAIL: DcR1 [20] and DcR2 [21], transmembrane receptors which lack functional death domains, and osteoprotegerin [22], a soluble molecule capable of binding to TRAIL. Consequently, a perturbation in the ratio of TRAIL decoy and death receptors is implicated in determining the responsiveness of cells to TRAIL. Furthermore, susceptibility to TRAIL-induced apoptosis can also be regulated by the levels of intracellular anti-apoptotic molecules, such as cellular FLICE-like inhibitory protein (cFLIP) and X-linked inhibitor of apoptosis proteins (XIAP). The role of c-FLIP in abrogating TRAIL-mediated apoptosis is due to its structural similarity to caspase-8. Indeed, by blocking the autoproteolytic cleavage of procaspase- 8 cFLIP halts the transduction of death signal, and high levels of cFLIP lead to lack of sensitivity to TRAIL in several cancer types [23-27]. Moreover, XIAP which is the most potent inhibitor of apoptosis proteins among the IAPs can bind and inactivate caspase-3, -7, and -9 [28], and high XIAP expression has been postulated as a mechanism of resistance to TRAIL apoptotic effect in different cancer cells [29-32]. (shortened).

At present, very few evidences have been published about the role of TRAIL on normal OBs demonstrating that these cells express all TRAIL receptors and are resistant to its apoptotic effect [13]. Moreover, TRAIL $-/-$ deficient mice do not show overt differences in gross density of bones [33]. Therefore, on the bases of literature findings it emerges that the effect of TRAIL on osteoblastic cells, either normal or transformed, could be more complex than it appears and becomes critically relevant to take into account the profound functional changes to which OBs undergo during their differentiation. Thus, in this study we investigated whether the sensitivity of OBs to TRAILmediated apoptosis could vary during their differentiation process and we also analyzed the mechanism/s responsible of TRAIL effect in undifferentiated and differentiated normal human OBs.

\section{Materials and Methods}

\section{Ethics Statement}

This study was approved by the Ethical Committee of Tor Vergata University Hospital (Protocol number 121/06 of 20 December 2006). All study participants provided written informed consent.

\section{Human OBs}

Trabecular bone specimens, obtained from healthy subjects, who undergo to femur surgery following traumatological events were provided by the Orthopaedic Department of Tor Vergata. These specimens were cleaned off soft tissues, reduced to small fragments, and digested with $0.5 \mathrm{mg} / \mathrm{mL}$ Clostridium histolyticum neutral collagenase (Sigma Chemical Co., St. Louis, MO, USA) in phosphate-buffered saline (PBS) with gentle agitation for $30 \mathrm{~min}$ at $37{ }^{\circ} \mathrm{C}$. Bone fragments were then washed (three times) with minimum essential medium ( $\alpha$-MEM) (Gibco Ltd., Uxbridge, UK) containing $3.024 \mathrm{~g} / \mathrm{L}$ sodium bicarbonate, and cultured in medium supplemented with $10 \%$ fetal calf serum (Gibco Ltd.), $100 \mathrm{IU} / \mathrm{mL}$ penicillin, $100 \mathrm{mg} / \mathrm{mL}$ streptomycin, $2.5 \mathrm{mg} / \mathrm{mL}$ amphotericin $\mathrm{B}$, and $50 \mathrm{IU} / \mathrm{mL}$ mycostatin, at $37{ }^{\circ} \mathrm{C}$ in a water-saturated atmosphere containing $5 \% \mathrm{CO}_{2}$. Cells were fed by medium replacement every 3-4 days. In these conditions, the osteoblast resident in the explant proliferated and migrated to the culture substrate, reaching confluence within 3-4 weeks. Cells were then trypsinized and transferred into appropriate culture dishes for characterization and experiments.

\section{Cell Viability Assay}

Mitochondrial dehydrogenases activity was determined by MTT-assay. This assay is based on the ability of formazan dye crystals to develop only in living cells, providing an indication of the mitochondrial integrity and activity 
which, in turn, may be interpreted as a measure of cell viability. Osteoblasts were cultured in 96-well tissue-culture plates; after having reached the confluence, part of the cells were stopped and used as Time $0\left(t_{0}\right)$ while the others were grown with the same medium supplemented with $50 \mu \mathrm{g} / \mathrm{mL}$ ascorbic acid and Dexametasone $10^{-8} \mathrm{M}$ for 3, 7,10 , and 20 days $\left(t_{3}, t_{7}, t_{10}\right.$, and $\left.t_{20}\right)$ to obtain differentiation. Cells were treated with different concentrations of rh-TRAIL (10-500 ng/mL, TRAIL/TNFSF10, R\&D Systems Inc., MN, USA) for 24 and $48 \mathrm{~h}$ to evaluate the cell viability, on days 0,10 , and 20 . The cell viability experiments were performed in the presence of $10 \%$ FBS. 3-(4,5-dimethylthiazol-2-yl)-2,5-diphenyltetrazolium bromide (MTT) $0.5 \mathrm{mg} / \mathrm{mL}$ were added to the culture media followed by $4 \mathrm{~h}$ incubation at $37{ }^{\circ} \mathrm{C}$ in a humidified $5 \%$ $\mathrm{CO}_{2}$ atmosphere. The reaction was stopped by adding $150 \mu \mathrm{L}$ of $0.04 \mathrm{~N} \mathrm{HCl}$ in absolute isopropanol. The optical density was read at $570 \mathrm{~nm}$ using an automatic plate reader (550 Microplate Reader Bio-Rad Laboratories Inc., CA, USA). The results were compared to cells incubated under control conditions. In parallel, the effect of $100 \mathrm{ng} / \mathrm{mL}$ TRAIL on OBs viability was evaluated in cells pretreated for $30 \mathrm{~min}$ with $5 \mu \mathrm{g} / \mathrm{mL}$ of antagonist anti-DR5 or antiDR4 neutralizing monoclonal antibodies (mAbs) (Alexis, San Diego, CA, USA) or an irrelevant anti-IgG.

\section{DNA Fragmentation}

Human OBs were cultured in 6-well tissue-culture plates until reaching the confluence in $\alpha$-MEM supplemented with $10 \%$. DNA extracts were prepared from OBs treated with rh-TRAIL at a concentration of $100 \mathrm{ng} / \mathrm{mL}$ for different times. DNA was purified using the Apoptotic DNA Ladder Kit (Roche Applied Science, Mannheim, Germany), to detect the typical apoptotic DNA fragments (ladder), according to the manufacturer's instructions. The kit provides a lyophilized apoptotic U937 cell sample as positive control. Samples were then electrophoresed on $2 \%$ agarose gel containing $0.01 \%$ ethidium bromide, and the resulting bands were detected by a light sensitive CCD video system (BioDocAnalyze, Whatman Biometra, Göttingen, Germany).

\section{RNA Isolation and Real-Time PCR Analysis}

OBs were seeded in 6-well tissue-culture plates; after having reached the confluence, part of the cells were stopped and used as Time 0 while the others were grown with the same medium supplemented with $50 \mu \mathrm{g} / \mathrm{mL}$ ascorbic acid and Dexametasone $10^{-8} \mathrm{M}$ for $3,7,10$, and 20 days to obtain differentiation. At the indicated times, OB cultures were subjected to mRNA extraction using spin columns (RNeasy, Qiagen, Hilden, Germany) to detect the expression of cFLIP, XIAP, and the housekeeping genes glyceraldehyde phosphate dehydrogenase (GAPDH). Firststrand cDNA was generated using the SuperScript FirstStrand Synthesis System kit for RT-PCR (Invitrogen, Carlsbad, CA, USA). Briefly for the first-strand cDNA synthesis, an RT mixture containing $1 \mu \mathrm{g}$ total RNA, dNTPs, Oligo(dT), RT buffer, $\mathrm{MgCl}_{2}$, DTT, RNaseOUT, SuperScript II RT, DEPC-treated water to final volume $100 \mu \mathrm{L}$ was prepared, according to the manufacturer's instructions. cDNA was amplified with the iTaq SYBR Green supermix with ROX kit (Bio-Rad Laboratories, BioRad Laboratories Inc., CA, USA), and the PCR amplification was performed using the Chromo4 Real-Time PCR Detection System (Bio-Rad Laboratories). The following primer pairs were used for the PCR amplification: cFLIP (S: 5'-AAGCTGTCTGTCGGGGACTT-3'; AS: 5'-GGTG GGTCTCCACAGCTTTT-3'); XIAP (S: 5'-GACAGTATG CAAGATGAGTCAAGTCA-3'; AS: 5'-GCAAAGCTTCC TCCTCTTGCAG-3'); osteocalcin (S: 5'-ACACTCCTCG CCCTATTG-3'; AS: 5'-CAGCCATTGATACAGGTAG C-3'); alkaline phosphatase (Ph Alk) (S: 5'-CGCACGG AACTCCTGACC-3 ${ }^{\prime}$; AS: 5'-GCCACCACCACCATC TCG-3'); osteopontin (S: 5'-CTGATGAATCTGATGAA CTGGTC-3'; AS: 5'-GTGATGTCCTCGTCTGTAGC-3'); GAPDH (S: TCATCCCTGCCTCTACTG; AS: TGCTTC ACCACCTTCTTG). The running conditions were incubation at $95^{\circ} \mathrm{C}$ for $3 \mathrm{~min}$, and 40 cycles of incubation at $95{ }^{\circ} \mathrm{C}$ for $15 \mathrm{~s}$ and $60{ }^{\circ} \mathrm{C}$ for $30 \mathrm{~s}$. After the last cycle, the melting curve analysis was performed into $55-95{ }^{\circ} \mathrm{C}$ interval by incrementing the temperature of $0.5^{\circ} \mathrm{C}$. The fold change values were calculated by Pfaffl method [34].

\section{Western Blot Analysis}

To study the expression of TRAIL-Rs during OB differentiation, cells were seeded in 6-well tissue-culture plates; after having reached the confluence, part of the cells were lysed and used as Time 0 while the others were grown with the same medium supplemented with $50 \mu \mathrm{g} / \mathrm{mL}$ ascorbic acid and Dexametasone $10^{-8} \mathrm{M}$ for 3, 7, 10, and 20 days to obtain differentiation. At the indicated times, OBs were lysed by incubation on ice for $30 \mathrm{~min}$ in lysis buffer containing $50 \mathrm{mmol} / \mathrm{L}$ Tris- $\mathrm{HCl}(\mathrm{pH} \mathrm{8.0)}, 150 \mathrm{mmol} / \mathrm{L} \mathrm{NaCl}$, $5 \mathrm{mmol} / \mathrm{L}$ ethylenediaminetetraacetic acid, $1 \% \mathrm{NP} 40$, and $1 \mathrm{mmol} / \mathrm{L}$ phenylmethyl sulfonyl fluoride.

To study caspase- 8 and -3 activation, OBs were seeded in 6-well tissue-culture plates; after having reached the confluence, the cells were treated with $100 \mathrm{ng} / \mathrm{mL}$ rhTRAIL for $0-8 \mathrm{~h}$. Cells were lysed by incubation on ice for $30 \mathrm{~min}$ in lysis buffer containing $20 \mathrm{mM}$ Tris- $\mathrm{HCl}(\mathrm{pH}$ 7.5), $1 \%$ Triton $\mathrm{X}-100,150 \mathrm{mM} \mathrm{NaCl}, 10 \%$ glycerol, $1 \mathrm{mM} \mathrm{Na} \mathrm{VO}_{4}, 50 \mathrm{mM} \mathrm{NaF}, 100 \mathrm{mM}$ phenylmethylsulfonyl fluoride, and a commercial protease inhibitor mixture 
(Sigma Aldrich). About $30 \mu \mathrm{g}$ of cell proteins was subjected to $12 \%$ SDS-PAGE gel and subsequently transferred to nitrocellulose membranes (Hybond, Amersham Pharmacia, London, UK). The blots were probed overnight at $4{ }^{\circ} \mathrm{C}$ with mouse anti-caspase 3 (Cell Signaling, San Diego, CA, USA) and anti- $\beta$-actin monoclonal Abs (Chemicon International Inc.), rabbit anti-DR4, anti-DR5, anti-DcR1, anti-DcR2 (Abcam, Cambridge, UK), anticaspase 8 (BD Pharmingen, San Diego, CA, USA), anticFLIP and anti-XIAP (Cell Signaling Technology, Danvers, MA, USA) polyclonal Abs. After incubation with the appropriate fluorescent-dye-conjugated secondary Ab (LICOR Biosciences GmbH Bad Homburg, Germany), specific reactions were revealed with the LI-COR's Odyssey Infrared Imaging System (LI-COR Biosciences-Biotechnology, Lincoln, NE, USA). Densitometric analysis was performed using LI-COR's Odyssey Infrared Imaging System Application Software version 3.0 (LI-COR Biosciences-Biotechnology).

\section{Statistical Analyses}

Statistical analyses were performed by Student's $t$ test with the Statistical Package for the Social Sciences (spssx/pc) software (SPSS, Chicago, IL, USA). A value of $p<0.05$ was taken to be statistically significant.

\section{Results}

Sensitivity of OBs to TRAIL-Mediated Apoptosis

Sensitivity to TRAIL in human OBs was investigated by analyzing cell viability through MTT-assay in undifferentiated and differentiated OBs in the presence of TRAIL. OBs were cultured in 96-well tissue-culture plates; after the cells reached the confluence, part of them have been used as undifferentiated cells $\left(t_{0} \mathrm{OBs}\right)$ while the others were grown with osteogenic medium for 3 ( $\left.t_{3} \mathrm{OBs}\right), 7$ ( $\left.t_{7} \mathrm{OBs}\right), 10\left(t_{10}\right.$ OBs), and 20 ( $t_{20}$ OBs) days to induce their osteoblastic differentiation. All the cells were first characterized for their osteoblastic parameters (alkaline phosphatase, osteocalcin, and osteopontin), which were weakly expressed at $t_{0}$, slightly increased at $t_{3}$, and significantly enhanced at $t_{7}, t_{10}$, and $t_{20}$ (Supplementary Fig. 1 shows the data of $t_{0}, t_{10}$, and $t_{20}$ ). All the OBs were treated with increasing concentrations of rh-TRAIL (ranging from 10 to $500 \mathrm{ng} / \mathrm{mL}$ ) for 24 and $48 \mathrm{~h}$, and the viability of OBs was determined in both TRAIL-treated and untreated cells as control. As shown in Fig. 1a, the viability of $t_{0}$ OBs was reduced by TRAIL in a dose- and time-dependent manner. In particular, when OBs were exposed to $10 \mathrm{ng} / \mathrm{mL}$ rh-TRAIL for $24 \mathrm{~h}$ their viability was slightly but significantly decreased compared to untreated OBs. TRAIL treatment further reduced the viability of $t_{0} \mathrm{OBs}$ at 25 and $50 \mathrm{ng} / \mathrm{mL}$, the maximum decrease was reached at $100 \mathrm{ng} / \mathrm{mL}$ TRAIL, and no additional decline was observed in the presence of higher concentrations of the molecule. After $48 \mathrm{~h}$ TRAIL still induced a dose- and time-dependent decrease of $t_{0}$ OB viability, but the effect was more pronounced with respect to what was observed at $24 \mathrm{~h}$. In fact, the reduction of cell viability in the presence of $100 \mathrm{ng} / \mathrm{mL}$ TRAIL for $24 \mathrm{~h}$ was of about $30 \%$, while the same concentration after $48 \mathrm{~h}$ induced a decrease of about $48 \%$ with respect to the control (Fig. 1a). Surprisingly, we found that TRAIL fails to induce any effect on cell viability of differentiated OBs, either for early $\left(t_{3}\right.$ and $t_{7}$ OBs, data not shown) or late differentiated cells ( $t_{10}$ and $t_{20}$ OBs) after both 24 and $48 \mathrm{~h}$ TRAIL treatment with respect to untreated cells (Fig. 1b, c).

\section{Expression of TRAIL-Receptors During Osteoblast Differentiation}

On the basis of the described results, we investigated whether the $\mathrm{OB}$ differentiation process could induce a different expression of death and decoy TRAIL receptors. We found that undifferentiated and differentiated OBs express all TRAIL receptors at protein levels. In particular, the expression of the decoy receptor DcR2 was low in $t_{0}$ OBs, and increased in a time-dependent manner during the differentiation process, while the levels of DcR1 were not modified (Fig. 2). In addition, the expression of both death TRAIL receptors, DR4 and DR5, did not display any significant variation during the OB differentiation (Fig. 2). These findings indicated that only the variation of DcR2 expression took place during OB differentiation. Consequently, the ratio between death and decoy TRAIL receptor was in favor of the death receptors in undifferentiated OBs, thereby contributing to their sensitivity to TRAIL effect in reducing their cell viability. Differently, the ratio was shifted in favor of decoy receptors during and after OB differentiation, explaining the lack of TRAIL effect on the viability of these cells as shown in Fig. 1.

\section{DR5 Involvement in Osteoblast Apoptosis}

In order to identify which receptor could be involved in TRAIL-mediated effect on undifferentiated OBs, we studied the expression of death and decoy TRAIL receptors after cell treatment with $100 \mathrm{ng} / \mathrm{mL}$ TRAIL at different times (0-0.5-1-2-4-6-8 h). In Fig. 3, we showed that in undifferentiated OBs TRAIL treatment determined the reduction of DcR2 and the increase of DR5 expression. In particular, DcR2 levels significantly declined after $2 \mathrm{~h}$ TRAIL treatment, this reduction became more pronounced after $4 \mathrm{~h}$, and remained reduced compared to control up to 

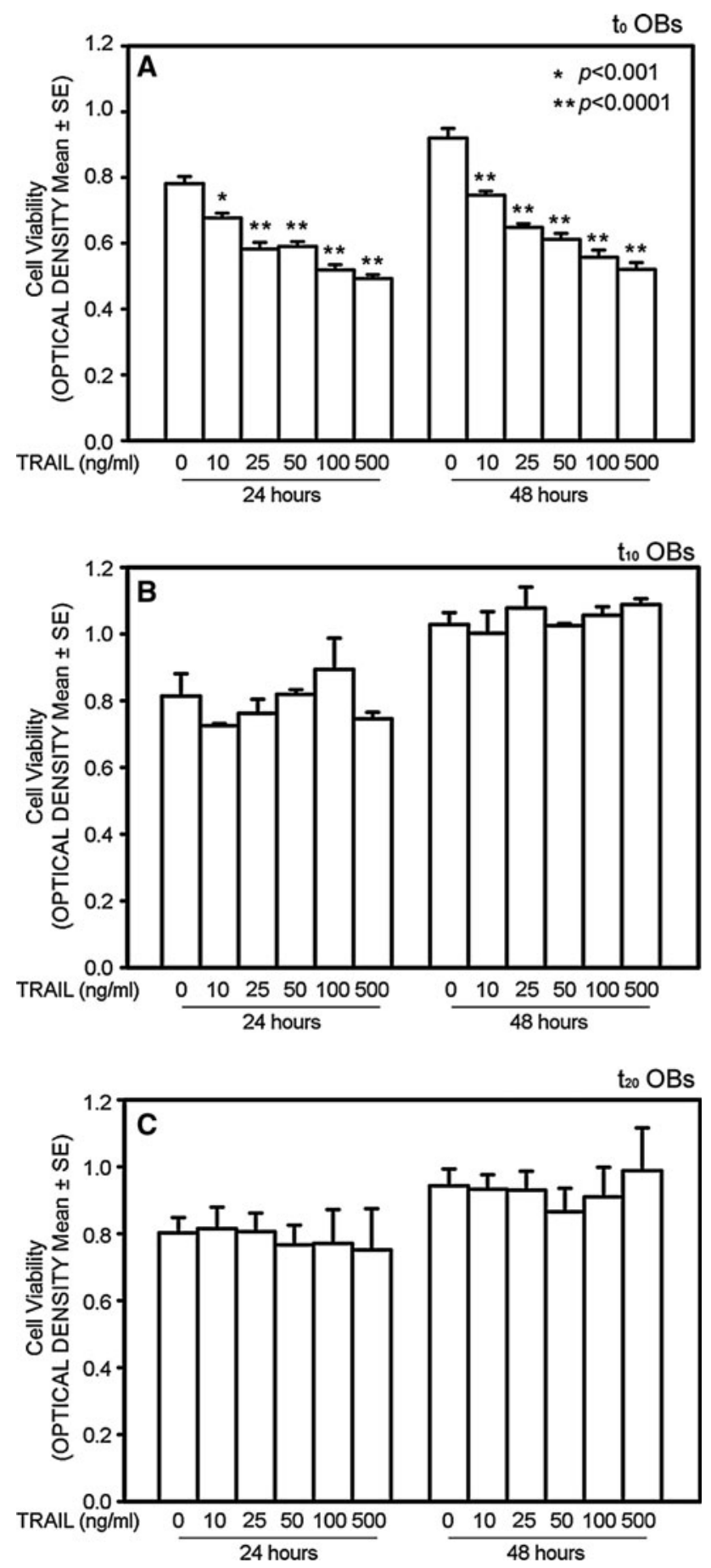

Fig. 1 Sensitivity of OBs to TRAIL-mediated apoptosis. Undifferentiated ( $\left.t_{0} \mathrm{OBs}\right)$ and differentiated $\mathrm{OBs}\left(t_{10}\right.$ and $\left.t_{20} \mathrm{OBs}\right)$, treated with increasing concentrations of rh-TRAIL (ranging from 10 to $500 \mathrm{ng} / \mathrm{mL}$ ) for 24 and $48 \mathrm{~h}$, were cultured in 96-well tissue-culture plates. a $t_{0}$ OB viability was reduced in a dose- and time-dependent manner by TRAIL treatment after both 24 and $48 \mathrm{~h}$. b, c The viability of both $t_{10}$ and $t_{20}$ OBs was not affected by the presence of TRAIL. The graphs represent the mean values \pm SE of optical density at $570 \mathrm{~nm}$ of five independent experiments in which each treatment was performed in quadruplicate
$8 \mathrm{~h}$ of treatment. On the contrary, the levels of DR5 increased after $1 \mathrm{~h}$ of treatment, reached the maximum levels after $2 \mathrm{~h}$ exposure to TRAIL, and remained higher up to $8 \mathrm{~h}$ treatment respect to DR5 baseline expression. In the same experiments, we demonstrated that TRAIL treatment did not modify the expression pattern of DcR1 and DR4 (data not shown). On the basis of these findings, we investigated $\mathrm{OB}$ viability in the simultaneous presence of TRAIL and anti-DR5 or anti-DR4 neutralizing antibodies or both. We demonstrated that the reduction of $t_{0}$ OB viability induced by TRAIL after $48 \mathrm{~h}$ was partially abolished only in the presence of anti-DR5 neutralizing antibody. No effect on the viability of TRAIL-treated OBs was exerted by anti-DR4 neutralizing antibody. Anti-DR4 or -DR5 as well as anti-IgG antibody was utilized as negative controls (Fig. 4).

\section{Expression of cFLIP and XIAP on OBs During Their Differentiation}

The described different sensitivity of undifferentiated and differentiated OBs to TRAIL lets us to study the different expression of the intracellular anti-apoptotic molecules cFLIP and XIAP during OB differentiation. By means of real-time PCR and western blot we showed that the expression levels of cFLIP increased during OB differentiation. In particular, the lowest mRNA levels of cFLIP were found in $t_{0} \mathrm{OBs}$; in comparison with these, cFLIP mRNA levels were unchanged in $t_{3}$ OBs, displayed 1.8and 2.8-fold change increase in $t_{7}$ OBs $(p<0.001)$ and $t_{10}$ OBs $(p<0.0001)$, respectively, and no further enhancement was detected until the $20^{\text {th }}$ day of cell differentiation $\left(t_{20} \mathrm{OBs}\right)$ (Fig. 5a). Instead, the gene expression of XIAP did not undergo significant changes during differentiation (Fig. 5b). The detection of protein levels of both cFLIP and XIAP overlaps the results of gene expression, in fact cFLIP increased during the differentiation process, while XIAP levels were not modified (Fig. 5c, d). Our results therefore demonstrate that differentiated OBs were protected from TRAIL-mediated apoptosis by the increase of cFLIP, which is an intracellular molecule inhibiting caspase-8.

TRAIL Activates Caspase-8 and -3 in Undifferentiated OBs

On the basis of the low expression of cFLIP in undifferentiated OBs, we investigated the activation of caspase- 8 and -3 .

As is well known, caspase- 8 is the initial caspase activated during TRAIL-induced apoptosis in other cell types [17-19]. Its activation consists of as first cleavage of 
Fig. 2 TRAIL-receptor expression during osteoblast differentiation. Western blot analysis has been performed on undifferentiated $\left(t_{0} \mathrm{OBs}\right)$ and differentiated OBs $\left(t_{3}, t_{7}, t_{10}\right.$, and $t_{20} \mathrm{OBs}$ ) to detect the expression of death and decoy TRAIL receptors. The histograms report the intensity of the bands quantified by densitometry and normalized to $\beta$-Actin. One of four independent experiments is also shown
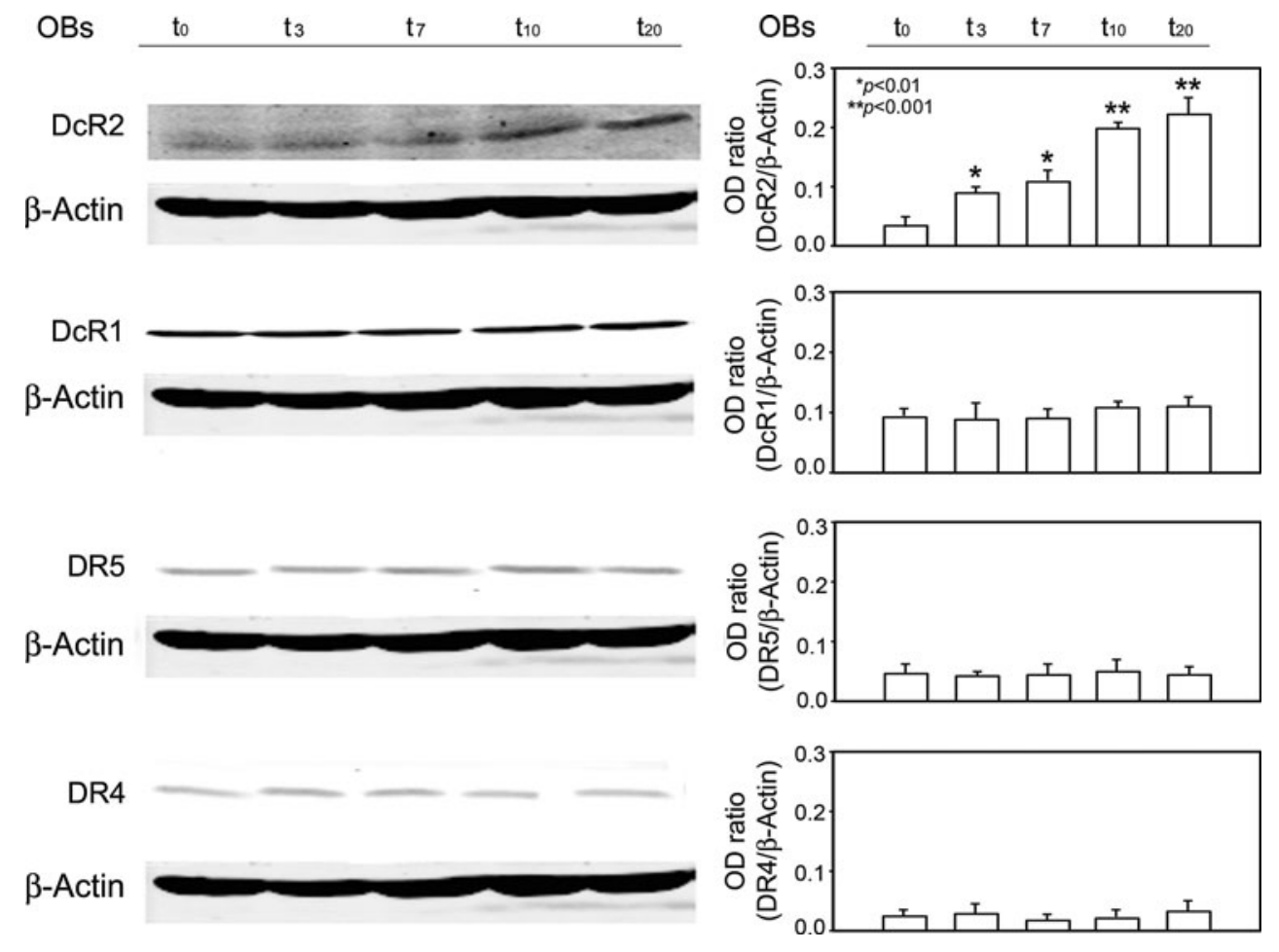
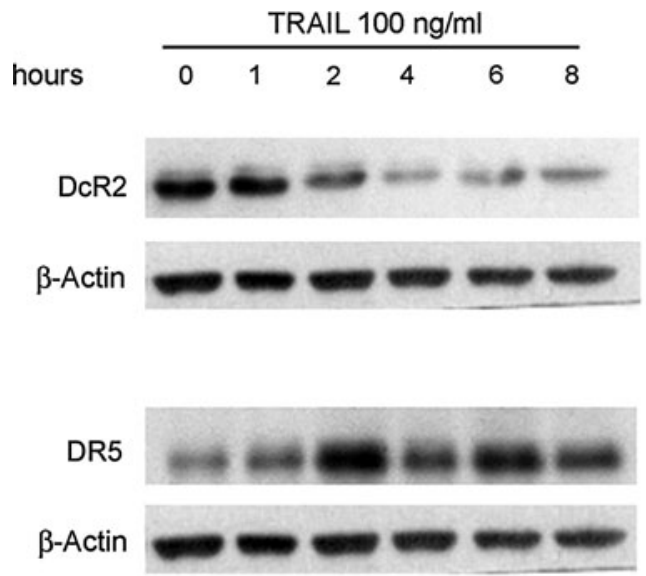

Fig. 3 TRAIL regulates DcR2 and DR5 expression in human undifferentiated OBs. Undifferentiated OBs, treated with $100 \mathrm{ng} /$ $\mathrm{mL}$ TRAIL at the indicated times, were lysed and analyzed by western blot analysis to detect the protein levels of TRAIL receptors.

procaspase- 8 which generates two small fragments, $\mathrm{p} 40$ and p36 intermediate forms, and a second cleavage resulting in the formation of the p23 active fragment. By western blot analysis, we demonstrated the activation of caspase- 8 in undifferentiated OBs treated with $100 \mathrm{ng} / \mathrm{mL}$ TRAIL from 0.5 up to $8 \mathrm{~h}$ treatment. In particular, the generation of intermediate forms (p40, p36) could be observed already after $2 \mathrm{~h}$ TRAIL treatment, and after $4 \mathrm{~h}$ a second cleavage took place, inducing formation of the p23 active caspase fragment (Fig. 6a). It is also known that

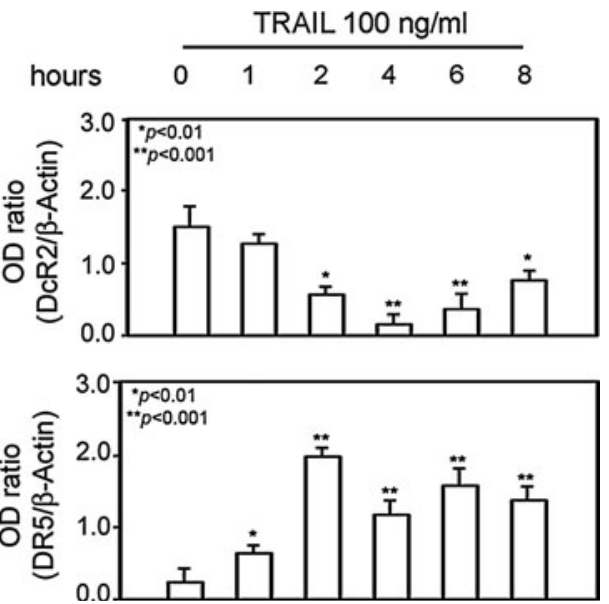

The expression of DcR2 and DR5 was modulated by TRAIL treatment. The intensity of the bands obtained by western blot analysis was quantified by densitometry (histogram) and normalized to $\beta$-Actin. One of five independent experiments is also shown

activated caspase- 8 cleaves caspase- 3 . Thus, we examined whether the cleavage of caspase- 3 could be induced by TRAIL treatment. As shown in Fig. 6b, the p17 cleaved form of caspase-3 was detected in undifferentiated OBs after 4, 6, and $8 \mathrm{~h}$ TRAIL treatment. The activation of caspases is followed by DNA fragmentation. In particular, following the addition of TRAIL to the culture media, the formation of the DNA ladder was clearly evident in OBs after 8 and $15 \mathrm{~h}$ incubation (data not shown). 


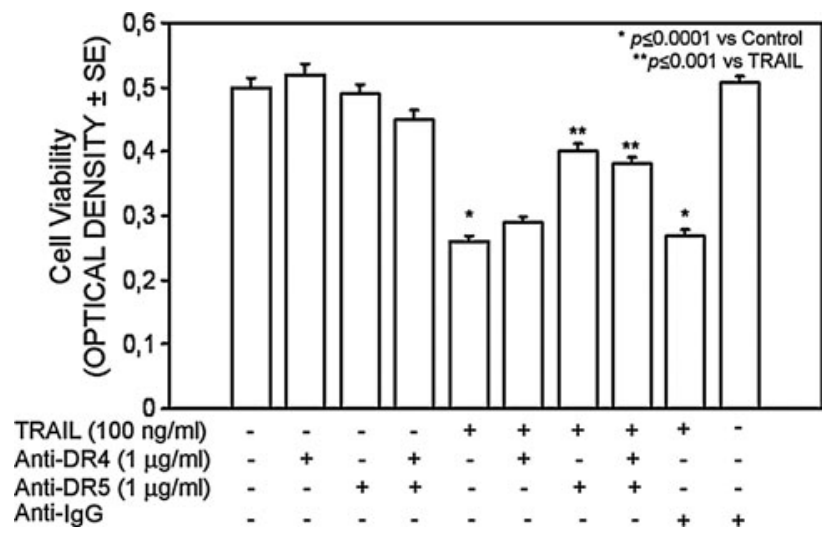

Fig. 4 Effect of neutralizing death receptor antibodies on osteoblast viability treated with TRAIL. Undifferentiated OBs were cultured in control conditions (-), in the presence of $100 \mathrm{ng} / \mathrm{mL}$ TRAIL (+), with $(+)$ or without $(-)$ anti-DR4, anti-DR5 neutralizing antibodies or anti-IgG control Ab, and the cell viability was assessed by MTTassay. OBs were pre-treated for $30 \mathrm{~min}$ with the indicated antibodies before the addition of TRAIL. The graph represents the mean values \pm SE of optical density at $570 \mathrm{~nm}$ of five independent experiments in which each treatment was performed in quadruplicate

\section{Discussion}

In this study, we investigated the effects of TRAIL on normal human OBs showing for the first time that their responsiveness to TRAIL varies during the differentiation
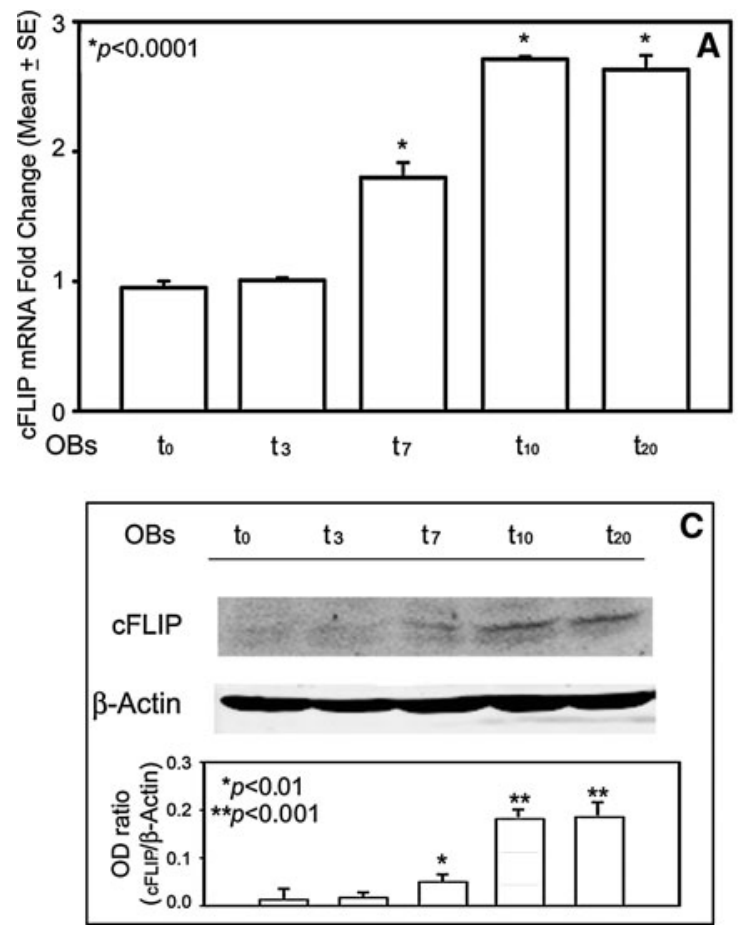

Fig. 5 Expression of cFLIP and XIAP on OBs during their differentiation. Undifferentiated ( $\left.t_{0} \mathrm{OBs}\right)$ and differentiated OBs $\left(t_{3}, t_{7}, t_{10}\right.$, and $t_{20} \mathrm{OBs}$ ), OBs were cultured in 6-well tissue-culture plates, and RNA extract and protein lysates were subjected to real-time PCR and process. We demonstrated that undifferentiated OBs are sensitive, whereas differentiated ones are resistant to TRAIL-apoptotic effect. Up to now, literature data have reported conflicting results concerning the apoptotic role of TRAIL in osteoblastic cells [8-14]. In particular, it has been demonstrated that some osteosarcoma cell lines are resistant to TRAIL apoptotic effect while others are sensitive $[8,11]$. In addition, their responsiveness to TRAIL could also change, this is the case of the BTK-143 osteogenic sarcoma cells, which can gradually acquire TRAIL resistance due to progressive acquisition of the DcR2 [12], or that of MG 63 osteosarcoma cells which undergo apoptosis in response to DR5 agonist [10]. Furthermore, other investigators have reported the resistance of OB-like cells from osteoarthritic patients or normal human OBs to TRAIL-mediated apoptotic effect [8, 13]. Differently, normal human OBs infected with Staphylococcus aureus or co-cultured with myeloma cells become sensitive to TRAIL-mediated apoptosis [13, 14].

The different behavior observed by the authors on osteoblastic cells in response to TRAIL could be related to the different models used in the mentioned studies, and particularly the data obtained on normal human OBs, displaying resistance to TRAIL-induced apoptosis, did not consider the stage of OB differentiation. This is of particular importance since OBs undergo profound morphological and
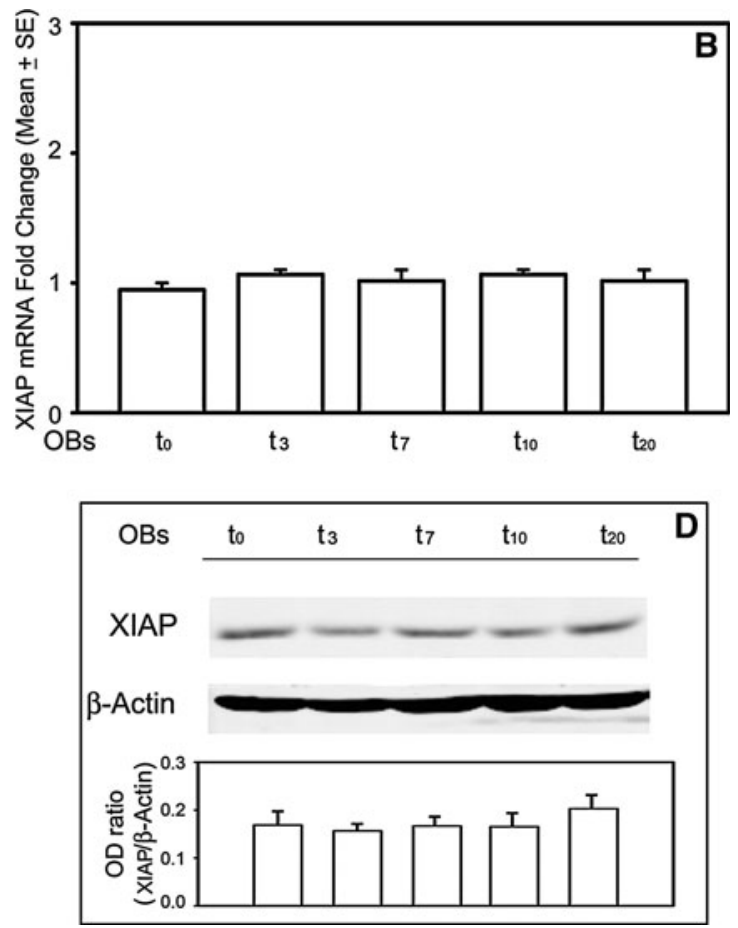

western blot analysis. Differential expression of cFLIP was demonstrated during the osteoblast differentiation at mRNA (a) and protein level $(\mathbf{c})$, while no changes were found in XIAP levels $(\mathbf{b}, \mathbf{d})$. One of four independent experiments is also shown 

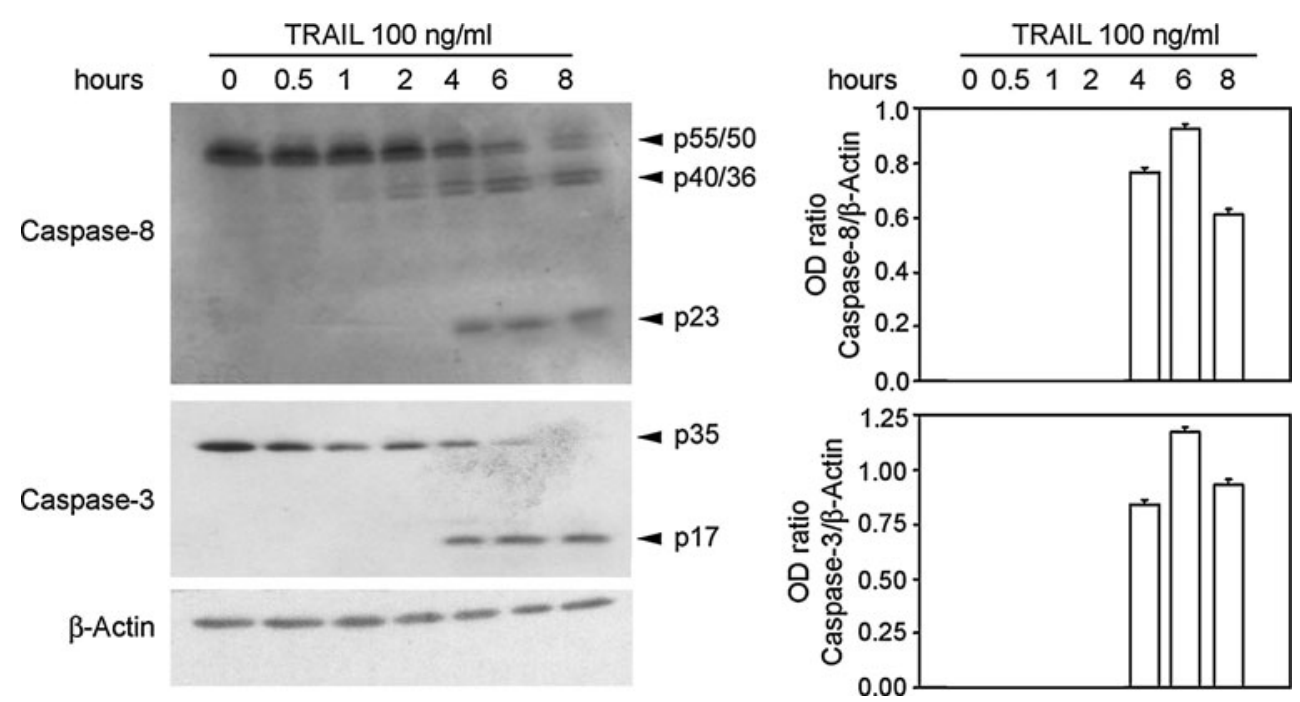

Fig. 6 TRAIL activates caspase- 8 and -3 in undifferentiated OBs. Undifferentiated OBs, treated with $100 \mathrm{ng} / \mathrm{mL}$ TRAIL, were lysed at the indicated times and analyzed by western blot analysis to detect the protein levels of inactive and active forms of caspase- 8 and -3 . The

functional changes during their differentiation process, starting from the commitment of osteoprogenitor cells, their differentiation into pre-OBs and finally in mature OBs [35]. Thus, it becomes very important to take into account in which stage of cell differentiation TRAIL effects are studied in normal OBs. For this reason, we investigated whether the induction of OB differentiation can determine by itself a different responsiveness of OBs to TRAIL treatment. Interestingly, we found that only undifferentiated normal human OBs are sensitive to TRAIL-induced apoptosis in a time- and dose-dependent manner; on the contrary, differentiated OBs become resistant as demonstrated by the lack of TRAIL effect on their viability. Furthermore, we showed that the different behavior of undifferentiated and differentiated OBs to TRAIL treatment is associated to the variation of the ratio between death and decoy TRAIL receptors, which is critical in determining the fate of the cells. Indeed, we found that the induction of OB differentiation induced the increase of the DcR2 expression respect to undifferentiated OBs, whereas DR5, DR4, and DcR1 expressions were not modified during the differentiation process. These findings indicated that in undifferentiated OBs the ratio of TRAIL receptors was shifted toward the deaths, making these cells sensitive to TRAIL-apoptotic effect. On the contrary, in differentiated OBs the receptor ratio was moved in favor of the decoys, thereby protecting these cells from TRAIL-mediated apoptosis. In addition, we demonstrated that undifferentiated OBs respond to TRAIL by further pushing the receptor ratio in favor of those of deaths with the upregulation of DR5 and the down-regulation of DcR2, suggesting that DR5 can be the main receptor by which TRAIL triggers the apoptotic effect. This was also sustained by the fragments at $23 \mathrm{kDa}$ and $17 \mathrm{kDa}$ represent the active cleaved form for caspase- 8 and -3 . The intensity of the bands obtained by western blot analysis was quantified by densitometry (histogram) and normalized to $\beta$-Actin. One of five independent experiments is also shown

demonstration that only anti-DR5 neutralizing antibody almost completely restores the TRAIL-induced reduction of OB viability. Our findings indicate that in physiological conditions OBs acquiring a different expression of DcR2 and DR5 receptors vary their sensitivity to TRAIL. This is particularly relevant if we consider literature data, showing that in pathologic osteoblastic cells lines the same receptors modulate cell responsiveness to TRAIL [10, 12].

However, cell-resistance or -sensitivity to TRAILmediated apoptosis is at least only in part determined by the balance of its death and decoy receptors, as it can also be correlated to the levels of some intracellular antiapoptotic molecules, such as cFLIP. Thus, we subsequently evaluated the expression of cFLIP during OB differentiation. Interestingly, we showed low cFLIP mRNA and protein levels in undifferentiated OBs and its higher expression in differentiated OBs, indicating that, besides the different receptor ratio, the sensitivity or resistance to TRAIL-apoptotic effect is also associated to the varied c-FLIP expression during OB differentiation process. Our findings are in line with those from other authors demonstrating that the expression of cFLIP was highest in the TRAIL-resistant melanomas, while low or undetectable in the TRAIL-sensitive melanomas [26], and data from other investigators showing that high levels of cFLIP lead to lack of sensitivity to TRAIL in several cancer types [23-27]. Moreover, according with the low levels of cFLIP in undifferentiated $\mathrm{OBs}$, we demonstrated in these cells the activation of caspase-8, the cleavage of caspase- 3 , and DNA fragmentation, which represent the intracellular events of the typical apoptotic pathway activated by TRAIL in other cells. 
In conclusion, our results highlight a different responsiveness of human OBs to TRAIL: sensitivity of undifferentiated OBs to TRAIL cytotoxic effect and resistance of fully differentiated ones. These findings could be of relevant importance in determining the correct number of working OBs which are needed to build new bone. Moreover, in pathological conditions, in which the overexpression of TRAIL has been demonstrated [36-41], our data suggest that TRAIL could contribute to the impairment of OB differentiation by targeting the apoptosis of OB precursors.

Acknowledgments The authors thank Ministero dell'Istruzione Università e Ricerca (ex $60 \%$ Grant to Maria Grano) and Agenzia Spaziale Italiana (ASI-OSMA Grant to Maria Grano).

\section{References}

1. Bu, R., Borysenko, C. W., Li, Y., Cao, L., Sabokbar, A., et al. (2003). Expression and function of TNF-family proteins and receptors in human osteoblasts. Bone, 33, 760-770.

2. Gronowicz, G. A., McCarthy, M. B., Zhang, H., \& Zhang, W. (2004). Insulin-like growth factor II induces apoptosis in osteoblasts. Bone, 35, 621-628.

3. Hill, N. M., Horne, J. G., \& Devane, P. A. (1999). Donor site morbidity in the iliac crest bone graft. Australian and New Zealand Journal of Surgery, 69, 726-728.

4. Jilka, R. L., Weinstein, R. S., Parfitt, A. M., \& Manolagas, S. C. (2007). Quantifying osteoblast and osteocyte apoptosis: Challenges and rewards. Journal of Bone and Mineral Research, 22, 1492-1501.

5. Weinstein, R. S., \& Manolagas, S. C. (2000). Apoptosis and osteoporosis. American Journal of Medicine, 108, 153-164.

6. Hatakeyama, S., Tomichi, N., Ohara-Nemoto, Y., \& Satoh, M. (2000). The immunohistochemical localization of Fas and Fas ligand in jaw bone and tooth germ of human fetuses. Calcified Tissue International, 66, 330-337.

7. Ozeki, N., Mogi, M., Nakamura, H., \& Togari, A. (2002). Differential expression of the Fas-Fas ligand system on cytokineinduced apoptotic cell death in mouse osteoblastic cells. Archives of Oral Biology, 47, 511-517.

8. Atkins, G. J., Bouralexis, S., Evdokiou, A., Hay, S., Labrinidis, A., et al. (2002). Human osteoblasts are resistant to Apo2L/ TRAIL-mediated apoptosis. Bone, 31, 448-456.

9. Young, A. B., Cooley, I. D., Chauhan, V. S., \& Marriott, I. (2011). Causative agents of osteomyelitis induce death domaincontaining TNF-related apoptosis-inducing ligand receptor expression on osteoblasts. Bone, 48, 857-863.

10. Locklin, R. M., Federici, E., Espina, B., Hulley, P. A., Russell, R. G., et al. (2007). Selective targeting of death receptor 5 circumvents resistance of MG-63 osteosarcoma cells to TRAIL-induced apoptosis. Molecular Cancer Therapeutics, 6, 3219-3228.

11. Evdokiou, A., Bouralexis, S., Atkins, G. J., Chai, F., Hay, S., et al. (2002). Chemotherapeutic agents sensitize osteogenic sarcoma cells, but not normal human bone cells, to Apo2L/TRAILinduced apoptosis. International Journal of Cancer, 99, 491-504.

12. Bouralexis, S., Findlay, D. M., Atkins, G. J., Labrinidis, A., Hay, S., et al. (2003). Progressive resistance of BTK-143 osteosarcoma cells to Apo2L/TRAIL-induced apoptosis is mediated by acquisition of DcR2/TRAIL-R4 expression: Resensitisation with chemotherapy. British Journal of Cancer, 89, 206-214.
13. Alexander, E. H., Rivera, F. A., Marriott, I., Anguita, J., Bost, K. L., et al. (2003). Staphylococcus aureus-induced tumor necrosis factor-related apoptosis-inducing ligand expression mediates apoptosis and caspase- 8 activation in infected osteoblasts. BMC Microbiology, 3, 5.

14. Tinhofer, I., Biedermann, R., Krismer, M., Crazzolara, R., \& Greil, R. (2006). A role of TRAIL in killing osteoblasts by myeloma cells. FASEB Journal, 20, 759-761.

15. Pan, G., O'Rourke, K., Chinnaiyan, A. M., Gentz, R., Ebner, R., et al. (1997). The receptor for the cytotoxic ligand TRAIL. Science, 276, 111-113.

16. Sheridan, J. P., Marsters, S. A., Pitti, R. M., Gurney, A., Skubatch, M., et al. (1997). Control of TRAIL-induced apoptosis by a family of signaling and decoy receptors. Science, 277, 818-821.

17. Chaudhary, P. M., Eby, M., Jasmin, A., Bookwalter, A., Murray, J., et al. (1997). Death receptor 5, a new member of the TNFR family, and DR4 induce FADD dependent apoptosis and activate the NF-kappaB pathway. Immunity, 7, 821-830.

18. Nagata, S. (1997). Apoptosis by death factor. Cell, 88, 355-365.

19. Ashkenazi, A., \& Dixit, V. M. (1998). Death receptors: Signaling and modulation. Science, 281, 305-308.

20. Degli-Esposti, M. A., Smolak, P. J., Walczak, H., Waugh, J., Huang, C. P., et al. (1997). Cloning and characterization of TRAIL-R3, a novel member of the emerging TRAIL receptor family. Journal of Experimental Medicine, 186, 1165-1170.

21. Pan, G., Ni, J., Yu, G., Wei, Y. F., \& Dixit, V. M. (1998). TRUNDD, a new member of the TRAIL receptor family that antagonizes TRAIL signalling. FEBS Letters, 424, 41-45.

22. Emery, J. G., McDonnell, P., Burke, M. B., Deen, K. C., Lyn, S., et al. (1998). Osteoprotegerin is a receptor for the cytotoxic ligand TRAIL. Journal of Biological Chemistry, 273, 14363-14367.

23. Wang, X., Chen, W., Zeng, W., Bai, L., Tesfaigzi, Y., et al. (2008). Akt-mediated eminent expression of c-FLIP and Mcl-1 confers acquired resistance to TRAIL-induced cytotoxicity to lung cancer cells. Molecular Cancer Therapeutics, 7, 1156-1163.

24. Guseva, N. V., Rokhlin, O. W., Taghiyev, A. F., \& Cohen, M. B. (2008). Unique resistance of breast carcinoma cell line T47D to TRAIL but not anti-Fas is linked to p43cFLIP(L). Breast Cancer Research and Treatment, 107, 349-357.

25. Dolcet, X., Llobet, D., Pallares, J., Rue, M., Comella, J. X., et al. (2005). FLIP is frequently expressed in endometrial carcinoma and has a role in resistance to TRAIL-induced apoptosis. Laboratory Investigation, 85, 885-894.

26. Geserick, P., Drewniok, C., Hupe, M., Haas, T. L., Diessenbacher, P., et al. (2008). Suppression of cFLIP is sufficient to sensitize human melanoma cells to TRAIL- and CD95L-mediated apoptosis. Oncogene, 27, 3211-3220.

27. Horak, P., Pils, D., Kaider, A., Pinter, A., Elandt, K., et al. (2005). Perturbation of the tumor necrosis factor-related apoptosisinducing ligand cascade in ovarian cancer: Overexpression of FLIPL and deregulation of the functional receptors DR4 and DR5. Clinical Cancer Research, 11, 8585-8591.

28. Deveraux, Q. L., Leo, E., Stennicke, H. R., Welsh, K., Salvesen, G. S., et al. (1999). Cleavage of human inhibitor of apoptosis protein XIAP results in fragments with distinct specificities for caspases. EMBO Journal, 18, 5242-5251.

29. Chawla-Sarkar, M., Bae, S. I., Reu, F. J., Jacobs, B. S., Lindner, D. J., et al. (2004). Downregulation of Bcl-2, FLIP or IAPs (XIAP and survivin) by siRNAs sensitizes resistant melanoma cells to Apo2L/TRAIL-induced apoptosis. Cell Death and Differentiation, 11, 915-923.

30. Ndozangue-Touriguine, O., Sebbagh, M., Merino, D., Micheau, O., Bertoglio, J., et al. (2008). A mitochondrial block and expression of XIAP lead to resistance to TRAIL-induced apoptosis during progression to metastasis of a colon carcinoma. Oncogene, 27, 6012-6022. 
31. Lee, T. J., Lee, J. T., Park, J. W., \& Kwon, T. K. (2006). Acquired TRAIL resistance in human breast cancer cells are caused by the sustained cFLIP(L) and XIAP protein levels and ERK activation. Biochemical and Biophysical Research Communications, 351, 1024-1030.

32. Lippa, M. S., Strockbine, L. D., Le, T. T., Branstetter, D. G., Strathdee, C. A., et al. (2007). Expression of anti-apoptotic factors modulates Apo2L/TRAIL resistance in colon carcinoma cells. Apoptosis, 12, 1465-1478.

33. Sedger, L. M., Glaccum, M. B., Schuh, J. C., Kanaly, S. T., Williamson, E., et al. (2002). Characterization of the in vivo function of TNF-alpha-related apoptosis-inducing ligand, TRAIL/Apo2L, using TRAIL/Apo2L gene-deficient mice. European Journal of Immunology, 32, 2246-2254.

34. Pfaffl, M. W. (2001). A new mathematical model for relative quantification in real-time RT-PCR. Nucleic Acids Research, 29, e45.

35. Long, F. (2011). Building strong bones: Molecular regulation of the osteoblast lineage. Nature Reviews Molecular Cell Biology, $13,27-38$.

36. Mori, G., Brunetti, G., Colucci, S., Oranger, A., Ciccolella, F., et al. (2009). Osteoblast apoptosis in periodontal disease: Role of TNF-related apoptosis-inducing ligand. International Journal of Immunopathology and Pharmacology, 22, 95-103.
37. Mori, G., Brunetti, G., Colucci, S., Ciccolella, F., Coricciati, M., et al. (2007). Alteration of activity and survival of osteoblasts obtained from human periodontitis patients: role of TRAIL. Journal of Biological Regulators and Homeostatic Agents, 21, 105-114.

38. Brunetti, G., Oranger, A., Mori, G., Sardone, F., Pignataro, P., et al. (2011). TRAIL effect on osteoclast formation in physiological and pathological conditions. Front Biosci (Elite Ed), 3, 1154-1161.

39. Hofbauer, L. C., Schoppet, M., Christ, M., Teichmann, J., \& Lange, U. (2006). Tumour necrosis factor-related apoptosisinducing ligand and osteoprotegerin serum levels in psoriatic arthritis. Rheumatology (Oxford), 45, 1218-1222.

40. Zai-Xing, Y., Yan, L., Hao, W., Ye, Z., Chang, L., et al. (2008). Preliminary clinical measurement of the expression of TNFrelated apoptosis inducing ligand in patients with ankylosing spondylitis. Journal of Clinical Laboratory Analysis, 22, 138-145.

41. Xie, Y. D., Jin, L., \& Yu, Q. W. (2007). The role of IFN-gamma, IL-10, IL-12 and TRAIL in sera and synovium fluids from patients with rheumatoid arthritis. Xi Bao Yu Fen Zi Mian Yi Xue Za Zhi., 23, 536-537. 